\title{
The paradox of improved antiretroviral therapy in HIV: potential for nutritional modulation?
}

\author{
Lisa J. Ware ${ }^{1 *}$, S. A. Wootton ${ }^{1}$, J. M. Morlese ${ }^{2}$, B. G. Gazzard ${ }^{2}$ and A. A. Jackson ${ }^{1}$ \\ ${ }^{1}$ Institute of Human Nutrition, Southampton General Hospital, Tremona Road, Southampton SO16 6YD, UK \\ ${ }^{2}$ St Stephens Centre, Chelsea and Westminster Hospital, Fulham Road, London, SW10 9NH, UK
}

\begin{abstract}
Chronic infection with HIV type 1 is associated with alterations in macronutrient metabolism, specifically elevated plasma lipids, glucose and reduced insulin sensitivity. These alterations are most severe in patients at the later stages of AIDS, indicating a relationship with disease progression. Recently, a metabolic syndrome, termed lipodystrophy, has been described in successfully-treated HIV patients in whom the altered macronutrient metabolism of HIV infection appears to be amplified markedly, with concurrent alterations in adipose tissue patterning. This syndrome presents a paradox, as before the development of highly-active antiretroviral therapy (HAART) the most severe perturbations in metabolism were observed in the sickest patients. Now, the patients that respond well to therapy are showing metabolic perturbations much greater than those seen before. The implications of this syndrome are that, whilst life expectancy may be increased by reducing viral load, there are concomitant increases in the risk of cardiovascular disease, diabetes and pancreatitis within this patient population. The aetiology of the syndrome remains unclear. In a collaborative trial with the Chelsea and Westminster Hospital in London we have used stable-isotope-labelled fatty acids to examine the hypothesis that treatment with HAART causes a delayed clearance of dietary lipid from the circulation, resulting in the retention of lipid within plasma and the downstream changes in insulin and glucose homeostasis. This hypothesis would indicate a role for low-fat diets, exercise and drugs that reduce plasma lipid or insulin resistance, in modulating the response to antiretroviral therapy in HIV infection.
\end{abstract}

\section{Lipodystrophy: HIV: Metabolism: Lipids: Stable isotopes}

In vivo nutrient partitioning is the key behind the efficient use of the nutrients present within the diet, so that the integration of exogenous and endogenous substrates allows the continued growth and renewal of the body at the tissue, cell and molecular levels. In situations where this nutrient partitioning is disturbed, an opportunity exists to study how this integrated system works, and to identify therapies to target specific parts of the system. Chronic infection presents such an opportunity, as HIV disease progression is known to be associated with alterations in the metabolic competence of macronutrient partitioning, along with profound changes in body composition, most notably wasting with reductions in both lean body mass and fat mass (Kotler \& Heymsfield, 1998). In the UK alone there have been approximately 55000 cases of HIV infection, with another seven new cases reported each day (Terrence Higgins Trust, 2001). The management of this disease has altered dramatically over the past 10 years, with the devel- opment of new antiretroviral drugs, a better understanding of the disease process, monitoring of drug resistance and better medications for the treatment of HIV-associated illnesses. In light of these changes the focus of HIV patient management has altered from treating immediate problems to concerns about longer-term health issues, due to increased life expectancy in this population. In order to combat the wasting classically associated with disease progression and poor clinical outcome, the traditional nutrition advice to a patient would be to maintain energy intake, probably through the use of energy-dense high-fat foods. However, it now appears that the combination of antiretroviral drugs used to treat HIV infection is associated with a further disturbance in metabolic competence, resulting in elevated plasma lipids, insulin and glucose, not dissimilar to that observed in non-insulin-dependent diabetes mellitus and syndrome $\mathrm{X}$. Thus, it is prudent to review the alterations in nutrient partitioning that occur

\footnotetext{
Abbreviations: HAART, highly-active antiretroviral therapy; LPL, lipoprotein lipase; NEFA, non-esterified fatty acids; NRTI, nucleoside reverse transcriptase inhibitor; TAG, triacylglycerols.

*Corresponding author: Lisa Ware, present address SHS International Ltd, 100 Wavertree Boulevard, Liverpool L7 9PT, UK, fax +44 (0)151 228 2650, emaillware@shsint.co.uk
} 
within this patient population, using evidence from detailed metabolic studies to determine both the most appropriate nutritional advice and the potential for nutritional or pharmaceutical intervention to modulate the side effects of these life-maintaining therapies. In using this model to better understand effective nutrient partitioning, some insight may be gained that will aid the management of many diseases where an impaired and inefficient nutrient partitioning appears to be the primary or secondary pathology.

\section{HIV infection and metabolic control}

Hypertriacylglycerolaemia, defined as a fasting plasma triacylglycerol (TAG) of $>2.3 \mathrm{mmol} / \mathrm{l}$ (Tikkanen, 1992), predominantly due to increased VLDL, is prevalent in AIDS and is positively correlated with the cytokine interferon- $\alpha$ (Grunfeld \& Feingold, 1991, 1992; Constans et al. 1994). Interferon- $\alpha$ also shows a positive correlation with decreased TAG clearance (Grunfeld et al. 1992) and increased fasting hepatic synthesis of fatty acids (Hellerstein et al. 1993). However, it is not known if interferon- $\alpha$ directly induces hypertriacylglycerolaemia or is a marker for the immune system and possibly other cytokines (Grunfeld \& Feingold, 1991). Some research groups have shown that a less profound hypertriacylglycerolaemia exists in asymptomatic HIV infection in comparison with healthy controls (Hellerstein et al. 1993). However, other researchers state that hypertriacylglycerolaemia is only present in the advanced symptomatic stages of infection (AIDS), and has an inverse correlation with CD4 T-cell count (Gomez-Sirvent et al. 1994; Christeff et al. 1995) and a positive correlation with $\beta 2$-macroglobulin and tumour necrosis factor $\alpha$ (Fernandez-Miranda et al. 1998). Dietary fish oil has been shown to alter the cytokines released by lymphocytes during HIV infection (Bell et al. 1996), although the implications of this change on lipid metabolism are unknown. Given the known roles of specific fatty acids in immune activation (Calder, 1996) and cytokine production (Endres, 1993; Endres et al. 1989; Meydani et al. 1991), there may be an opportunity to modulate both these metabolic perturbations and the immune response to chronic infection through nutritional intervention.

In vitro studies show that HIV destroys host T-cells via a reduced host phospholipid synthesis and an increased neutral lipid synthesis, producing changes in host-cell membrane permeability similar to the action of paramyxoviruses (Cloyd et al. 1991). However, the relative contribution of this process to the observed elevations in plasma TAG is unknown. In vivo studies and animal experiments suggest that the marked increases in plasma TAG concentration appear to be primarily due to a combination of: (a) a reduced clearance time of TAG from plasma due to a reduction in endothelial lipoprotein lipase (LPL) enzyme activity (Grunfeld et al. 1991); (b) an increase in hepatic fatty acid synthesis (lipogenesis; Hellerstein et al. 1993); (c) an increased mobilisation of free fatty acids from peripheral adipocytes by lipolysis (Feingold et al. 1990; Mulligan et al. 1993). Plasma non-esterified fatty acid (NEFA) concentration may be elevated or reduced compared with controls, depending on the stage of infection and the individual's nutritional status (Nunez \& Christeff, 1994). The oxidation of fatty acids is also increased in HIV infection (Hommes et al. 1991; Mulligan et al. 1993), although whether this increase is due to the virus, the elevated plasma TAG concentration or increased NEFA flux remains unclear. Plasma cholesterol (Grunfeld et al. 1992) and phospholipid (Klein et al. 1992) concentrations tend to be lower in HIV-infected subjects compared with healthy controls. Polyunsaturated fatty acids (PUFA) were found to decrease in erythrocyte membranes of AIDS patients, and a correlation was found with both elevated malondialdehyde concentration (the endproduct of lipid peroxidation) and a CD4 count of $<50$ cells $/ \mathrm{mm}^{3}$ (Constans et al. 1995). Reduced concentrations of antioxidants such as Se, vitamin A (Sappey et al. 1994; Constans et al. 1995), glutathione (Jahoor et al. 1999) and vitamin E (Pacht et al. 1997) have also been found in plasma of HIV-infected patients compared with healthy controls.

The mechanism by which HIV infection induces these alterations in lipid metabolism remains unknown. In addition, the further elevations of plasma TAG with the progression of HIV infection to symptomatic AIDS would suggest a positive relationship between the extent of viral burden and plasma TAG. However, in HIV-infected individuals with elevated viral load effective and rapid reduction in viraemia through administration of highlyactive antiretroviral therapy (HAART) has been shown, in some cases, to increase plasma TAG (Carr et al. 1998) and not to reduce lipids within the circulation, as would be expected.

\section{Antiretroviral therapy and the metabolic syndrome}

The development of this metabolic syndrome (termed lipodystrophy due to alterations in body fat distribution) in successfully-treated HIV patients creates a paradox. In these patients a combination of HAART is used to effectively reduce viral burden and allow partial immune reconstitution, resulting in a better prognosis for the patient in terms of their HIV disease (Gazzard et al. 1999). However, the metabolic complications associated with chronic infection appear to be exacerbated, resulting in much higher levels of plasma lipid, with reduced glucose tolerance and insulin sensitivity (Batterham et al. 2000; Carr \& Cooper, 2000), in addition to a loss of subcutaneous adipose tissue and increased visceral adiposity in some patients (Silva et al. 1998). These morphological and metabolic changes are reported in up to $84 \%$ of patients with long-term HAART use (Carr et al. 1999). The implications of these alterations, in conjunction with increased life expectancy, are the increasing risk of cardiovascular disease (Koppel, 2000; Mercie, 2000), diabetes (Meyer et al. 1998; Carr et al. 1999) and pancreatitis (Sullivan et al. 1998) within this patient population. Furthermore, patients with this change in body habitus develop a disinclination to continue therapy, which dramatically reduces life expectancy.

Many theories have been put forward to explain how these antiretroviral drugs could be directly responsible for the development of this metabolic syndrome, and there appear, currently, to be two primary schools of thought. The first theory is that the viral protease against which the 
protease inhibitor drugs are targeted shares an extent of structural homology with specific host proteins involved in adipocyte function (cis-retinoic acid binding protein) and lipid metabolism (lipoprotein receptor-related protein), and that these proteins are also targets of the protease inhibitor drugs (Carr et al. 1998, 1999). The second major theory is that the nucleoside reverse transcriptase inhibitor (NRTI) drugs are phosphorylated intracellularly to the nucleoside triphosphate form, and incorporated into mitochondrial DNA in the process of mitochondrial replication. Consequently, chain elongation is interrupted, due to the lack of the hydroxyl group on the synthetic nucleoside, resulting in reduced mitochondrial numbers and increased mutations and, therefore, reduced function and inappropriate oxidation of fatty acids and glucose (Brinkman et al. 1998, 1999). However, there have been no detailed metabolic studies conducted to support or refute either of the principal hypotheses. In addition, it also remains unclear whether the metabolic syndrome is entirely due to a direct effect of the antiretroviral drugs. There does appear to be a synergistic interaction between PI and other HAART drug classes (Carr \& Cooper, 2000; Mallal et al. 2000). It is unknown whether the metabolic changes (a) precede or result from the morphological alterations, (b) are a direct side effect of the drugs, or (c) are indirectly related to HAART and unmasked by reducing viral replication or promoting partial immune reconstitution. These abnormalities are unlikely to be a direct effect of HIV type 1, as the syndrome occurs in the presence of effective viral suppression and independently of any relationship with viral load. However, the primary changes with HAART initiation, as shown directly by studies in HIV-negative healthy subjects, appear to be elevated plasma lipids (Sullivan et al. 1998; Purnell et al. 1999) and reduced insulin sensitivity (Noor et al. 2001). These early metabolic changes were largely ignored in the HIV-positive population until the more obvious complex changes in fat distribution within the body were recognised. The disturbance in lipid metabolism may be so profound as to result in marked elevations in fasting plasma TAG concentration, sometimes in excess of $50 \mathrm{mmol} / \mathrm{l}$; values far greater than those observed in classical dyslipidaemias such as NIDDM (Taskinen, 1995).

\section{Lipid partitioning in healthy individuals}

In a healthy individual with good metabolic competence, consuming even fatty meals results in a minimal excursion of plasma TAG concentration during the postprandial period, due to effective clearance of the dietary lipid from the plasma compartment. Effective clearance is a balance between lipoprotein-TAG hydrolysis and peripheral or hepatic fatty acid uptake. In the circulation the TAG-rich lipoprotein is exposed to hydrolysis and release of the dietary TAG by the enzyme LPL, which is stimulated by insulin (Frayn et al. 1994). This enzyme is particularly abundant on the vascular endothelial surface of adipocytes and skeletal muscle cells, and is attached to the surface by a heparin stalk. Chylomicrons containing lipid from the diet have been shown in vitro to have a higher binding affinity for the LPL enzyme than VLDL (Xiang et al. 1999). Following LPL action the generated free fatty acids can be taken up by the adipocyte or skeletal muscle cell. This process is thought to be a carrier-mediated process involving specific fatty acid-binding proteins, fatty acid translocase and fatty acid transport protein (Turcott et al. 1997), and is known as entrapment (Fig. 1). The efficiency with which the cell entraps the fatty acids determines how much free fatty acid remains in the circulation. The fatty acids that are not taken up by the cell bind to the protein albumin in the circulation to become NEFAs. A lipoprotein remnant is also formed following LPL action. Fatty acids that are taken up by the cell can either be oxidised to produce energy, or re-esterified to TAG and stored for later use. The integration of the intracellular pathways of fatty acid metabolism with the mechanisms of fatty acid uptake and fatty acid generation through TAG hydrolysis remain unclear.

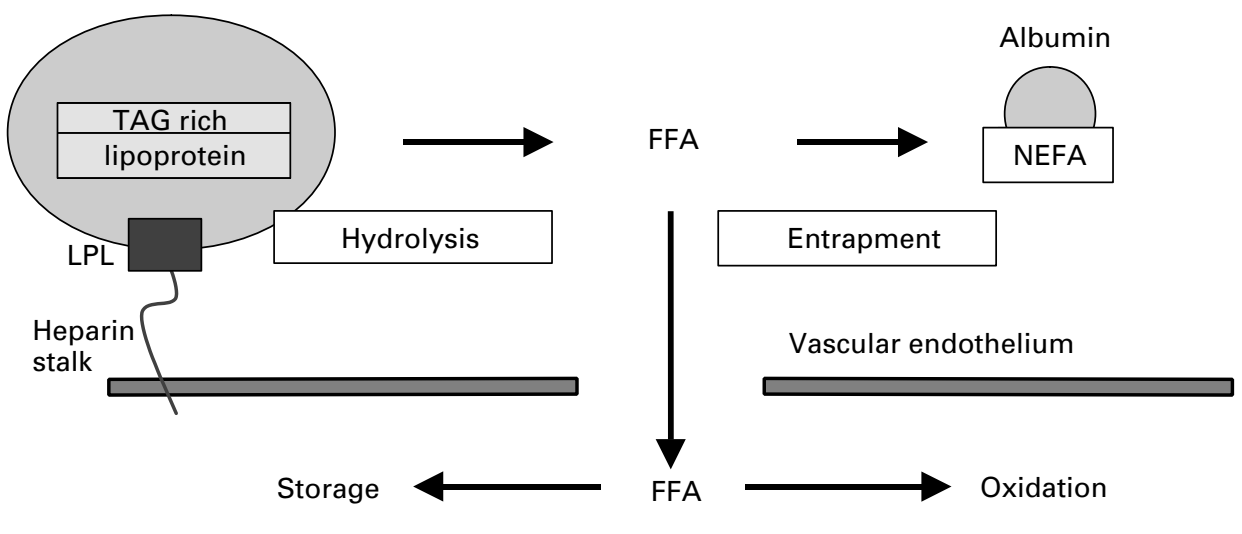

Adipocyte or skeletal muscle cell

Fig. 1. The integrated system of lipoprotein triacylglycerol hydrolysis, free fatty acid (FFA) generation and entrapment into the cell or release into the circulation bound to albumin as non-esterified fatty acid (NEFA). LPL, lipoprotein lipase. 
To what extent can disordered fatty acid uptake and metabolism cause the metabolic abnormalities?

An impaired coordinated control of TAG hydrolysis and fatty acid entrapment, possibly through a reduced action or sensitivity of the regulatory elements of this process such as insulin and the acylation-stimulating protein, can lead to less of the fatty acids derived from lipoprotein-TAG hydrolysis entering the cell and an increased flux of NEFA from the periphery to the liver (Fig. 2). Increased NEFA within the circulation would also result in decreased LPL activity by product inhibition, thereby reducing TAG clearance. Furthermore, increased flux of fatty acids to the liver would drive TAG synthesis and VLDL secretion, resulting in a re-secretion of the products of LPL hydrolysis of chylomicron-TAG back into the circulation as VLDL-TAG. This process would effectively provide another chance to clear the lipid from the circulation, but serve to maintain circulating TAG levels.

(a)

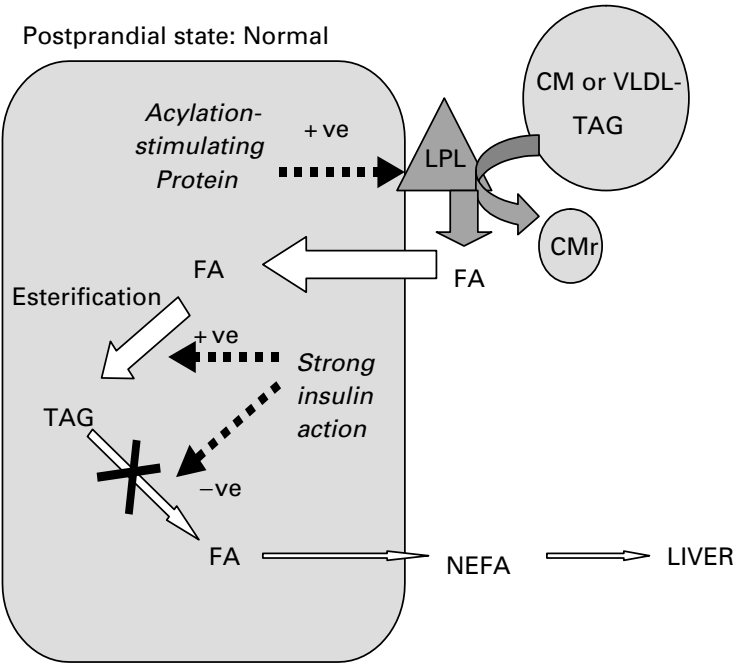

(b) Postprandial state: HIV+HAART

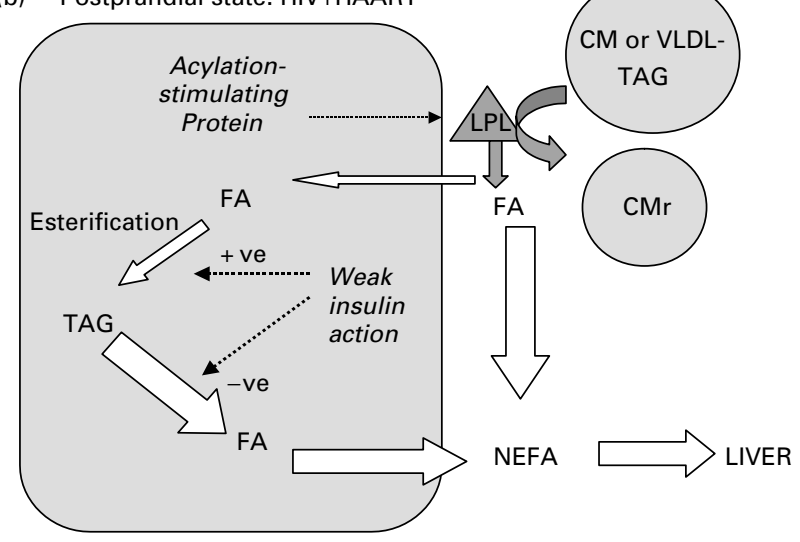

Fig. 2. The proposed mechanisms underlying dyslipidaemia in highly-active antiretroviral therapy (HAART)-associated lipodystrophy. FA, fatty acids; TAG, triacylglycerols; NEFA, non-esterified fatty acids; LPL, lipoprotein lipase; CM, chylomicron; CMr, chylomicron remnant; +ve, stimulated; -ve, inhibited.

\section{Our contribution}

Alterations in the metabolic competence to handle macronutrients may be better elucidated when the system is stressed, such as in the postprandial state following a meal when there is an influx of macronutrients into the circulation, rather than by simply studying fasting metabolism. Using stable-isotope-labelled fatty acid ([1-13 C]palmitic acid) as part of a test meal, we have examined postprandial lipid metabolism in HIV-infected males before any antiretroviral therapy (Ware et al. 2001). The results demonstrated that while the appearance of label in the plasma TAG pool was similar to that of HIV-negative healthy males of similar age, indicating no impairment in TAG hydrolysis, there were lower concentrations of label recovered from plasma NEFA and on breath as ${ }^{13} \mathrm{CO}_{2}$, possibly indicating altered metabolic demands for dietary fatty acids in the presence of chronic infection. We repeated this study in HIV-infected males with lipodystrophy associated with (1) NRTI drugs exclusively and (2) NRTI plus protease inhibitor combination antiretroviral therapy (Ware et al. 2000). The results demonstrated that while NRTI related lipodystrophy was associated with elevated fasting and postprandial plasma TAG concentration, lipodystrophy occurring with combined therapy produced typically much higher levels of plasma TAG, with elevated fasting and postprandial NEFA. These findings again suggest a synergistic effect of these two drug classes. While there was an increased retention of the label within the TAG pool in the combined therapy group, indicating reduced lipoprotein-TAG hydrolysis, there appeared to be an increased retention of label in the NEFA pool in the NRTI-related lipodystrophy group, indicating poor entrapment. These findings suggest that the two drug classes have independent effects. In addition, the recovery of label on breath as ${ }^{13} \mathrm{CO}_{2}$ in the NRTI-related lipodystrophy group was not different from that observed in the healthy nonHIV-infected males, indicating normal mitochondrial function for age in this group.

These results would suggest that a delayed clearance of dietary lipid from plasma occurs in lipodystrophy, with a reduced lipoprotein-TAG hydrolysis associated with protease inhibitor-containing therapy and increased NEFA flux associated with NRTI therapy. In another stable-isotope study (RV Sekhar, AC White, F Jahoor, F Visnegarwala, PJ Reeds and B Balasubramanyam, unpublished results) have also reported preliminary observations of elevated fatty acid turnover (whole-body rates of lipolysis, re-esterification and fatty acid oxidation) in patients with lipodystrophy compared with HIV-negative controls. In addition to the metabolic alterations associated with impaired fatty acid metabolism, a reduced ability to take up fatty acids specifically in peripheral tissues may result in the subcutaneous fat wasting, driving TAG storage to take place in visceral deposits and creating the morphological alterations observed in lipodystrophy.

If this process is responsible for the early development of the metabolic syndrome, it may be possible to modulate this process through low-fat diets (GJ Moyle, M Lloyd, B Reynolds and C Baldwin, unpublished results), specifically when combined with increased exercise regimens 
(Roubenouff et al. 1999; Yarasheski et al. 2000) to promote fatty acid uptake in the periphery through increased insulin sensitivity. There may also be a role for insulin sensitisers such as metformin, or plasma lipid-lowering drugs such as fibrates, in the treatment and possibly prevention of the metabolic syndrome associated with HAART use. However, until the pathophysiology of this metabolic syndrome has been uncovered, the recommendation to begin low-fat highcarbohydrate diets should not be made, as this regimen could exacerbate the situation if reduced insulin sensitivity is the primary event.

\section{Summary}

More detailed metabolic studies are required to determine the cause of this fatty acid metabolic dysregulation. It is still unclear whether the drugs used in HAART are directly responsible for these changes, although evidence does point towards independent effects of the different drug classes, resulting in combined synergistic effects on lipid metabolism. It is becoming increasingly clear that nutrition in HIV-positive individuals is now more than a weightmaintenance issue, and that the balance of macronutrients and micronutrients within the diet may modulate both the disease process and possibly the response to the lifemaintaining antiretroviral therapies.

\section{Acknowledgements}

This work was conducted predominantly at the Chelsea and Westminster Hospital, London and the authors would like to thank the staff both in London and Southampton for their support, particularly Dr G. C. Burdge and Dr A. E. Jones for their technical contribution, and all the patients that gave their time so generously to participate in these trials.

\section{References}

Batterham M, Garsia R \& Greenop P (2000) Dietary intake, serum lipids, insulin resistance and body composition in the era of HAART 'Diet FRS Study'. AIDS 14, 1839-1843.

Bell SJ, Chavali S, Bistrian B, Connolly CA, Utsonomiya T \& Forse RA (1996) Dietary fish oil and cytokine production during HIV infection. Journal of Parenteral and Enteral Nutrition 20, 43-49.

Brinkman K, Hofstede H, Burger D, Smeitinik J \& Koopmans P (1998) Adverse effects of reverse transcriptase inhibitors: mitochondrial toxicity as common pathway. AIDS 12, 1735-1744.

Brinkman K, Smeitinik J, Romjin J \& Reiss P (1999) Mitochondrial toxicity induced by nucleoside-analogue reverse transcriptase inhibitors is a key factor in the pathogenesis of antiretroviraltherapy-related lipodystrophy. Lancet 354, 1112-1115.

Calder PC (1996) Effects of fatty acids and dietary lipids on cells of the immune system. Proceedings of the Nutrition Society 55, $127-150$.

Carr A \& Cooper D (2000) Adverse effects of antiretroviral therapy. Lancet 356, 1423-1430.

Carr A, Samaras K, Chisholm D \& Cooper D (1998) Pathogenesis of HIV-1 protease inhibitor-associated peripheral lipodystrophy, hyperlipidaemia, and insulin resistance. Lancet 352, 1881-1883.
Carr A, Samaras K, Thorisdottir A, Kauffman G, Chisholm D \& Cooper D (1999) Diagnosis, prediction and natural course of HIV-1 protease-inhibitor-associated lipodystrophy, hyperlipidaemia, and diabetes mellitus: a cohort study. Lancet $\mathbf{3 5 3}$, 2093-2099.

Christeff N, Lortholary O, Casassus P, Thobie N, Dalle M, Veyssier P, Guillevin L \& Nunez E (1995) Serum lipid concentration with reference to the clinical and immunological status of HIV infected men. Annales de Médecine Interne 146, 490-495.

Cloyd MW (1991) Perturbation of host-cell membrane is a primary mechanism of HIV cytopathology. Virology 181, 500-511.

Constans J, Pellegrin J \& Peuchant E (1994) Plasma lipids in HIVinfected patients: a prospective study in 95 patients. European Journal of Clinical Investigation 24, 416-420.

Constans J, Peuchant E \& Pellegrin J (1995) Fatty acids and plasma antioxidants in HIV-positive patients: correlation with nutritional and immunological status. Clinical Biochemistry 28, 421-426.

Endres S (1993) Messengers and mediators: interactions among lipids, eicosanoids and cytokines. American Journal of Clinical Nutrition 57, 7895-8005.

Endres S, Ghorbani R, Kelley VE, Georgilis K, Lonnemann G, van der Meer JMW, Cannon JG, Rogers TS, Klempner MS, Weber PC, Schaeffer EJ, Wolff SM \& Dinarello CA (1989) The effect of dietary supplementation with $n-3$ polyunsaturated fatty acids on the synthesis of interleukin-1 and tumour necrosis factor by mononuclear cells. New England Journal of Medicine 320, 265-271.

Feingold K, Adi S, Staprins I, Moser A, Neese R, Verdier JA, Doerrler W \& Grunfeld C (1990) Diet affects the mechanisms by which TNF stimulates hepatic triglyceride production. American Journal of Physiology 259, E177-E184.

Fernandez-Miranda C, Pulido F \& Carrillo JL (1998) Lipoprotein alterations in patients with HIV infection: relation with cellular and humoral immune markers. Clinica Chimica Acta 274, 63-70.

Frayn K, Shadid S, Hamlani R, Humphreys S, Clark ML, Fielding BA, Boland O \& Coppack SW (1994) Regulation of fatty acid movement in human adipose tissue in the postabsorptive-topostprandial transition. American Journal of Physiology 266, E308-E317.

Gazzard B (editor) (1999) Weight loss and diarrhoea. In Chelsea and Westminster Hospital AIDS Care Handbook, pp. 201-214. London: Mediscript Ltd.

Gomez-Sirvent JL, Alonso-Socas MM, Santolaria-Fernandez FJ, Gonzalez-Reimers CE, Rodriguez-Moreno F \& EssardasDaryanani H (1994) Prognostic value of triglyceride levels in early HIV infection. AIDS 8, 1740-1741.

Grunfeld C \& Feingold K (1991) The metabolic effects of tumour necrosis factor and other cytokines. Biotherapy 3, 143-158.

Grunfeld C \& Feingold K (1992) Metabolic disturbances and wasting in AIDS. New England Journal of Medicine 327, 329-335.

Grunfeld C, Kotler D \& Shigenaga (1991) Circulating IFN-alpha and hypertriglyceridaemia in AIDS. American Journal of Medicine 90, 154-162.

Grunfeld C, Pang M, Doerrler W, Shigenaga J, Jensen P \& Feingold K (1992) Lipids, lipoproteins, triglyceride clearance, and cytokines in human immunodeficiency virus infection and the acquired immunodeficiency syndrome. Journal of Clinical Endocrinology and Metabolism 74, 1045-1052.

Hellerstein M, Grunfield C, Wu K, Christiansen M, Kaempfer S, Kletke C \& Shackleton C (1993) Increased de novo hepatic lipogenesis in HIV infection. Journal of Clinical Endocrinology and Metabolism 76, 559-565.

Hommes M, Romjin J, Endert E \& Sauerwein H (1991) Resting energy expenditure and substrate oxidation in HIV-infected 
asymptomatic men: HIV affects host metabolism in the early asymptomatic stage. American Journal of Clinical Nutrition 54, 311-315.

Jahoor F, Jackson AA, Gazzard B, Phillips G, Sharpstone D, Frazer M \& Heird W (1999) Erythrocyte glutathione deficiency in symptom-free HIV infection is associated with decreased synthesis rate. American Journal of Physiology 276, E205-E211.

Klein A, Bruser B, Bast M \& Rachlis A (1992) Progress of HIV infection and changes in the lipid membrane structure of CD4+ cells. AIDS 6, 332-333.

Koppel K (2000) Serum lipid levels associated with increased risk for cardiovascular disease is associated with highly active antiretroviral therapy (HAART) in HIV-1 infection. International Journal of STD and AIDS 11, 451-455.

Kotler D \& Heymsfield S (1998) HIV Infection: a model chronic illness for studying wasting diseases. American Journal of Clinical Nutrition 68, 519-520.

Mallal S, John M, Moore C, James I \& McKinnon E (2000) Contribution of nucleoside analogue reverse transcriptase inhibitors to subcutaneous fat wasting in patients with HIV infection. AIDS 14, 1309-1316.

Mercie P (2000) Atherogen lipid profile in HIV-1 infected patients with lipodystrophy syndrome. European Journal of Internal Medicine 11, 257-263.

Meydani SN, Endres S, Woods MM, Goldin BR, Soo C, MorrillLabrode A, Dinarello C \& Gorbach SL (1991) Oral (n-3) fatty acid supplementation suppresses cytokine production and lymphocyte proliferation: comparison between young and older women. Journal of Nutrition 121, 547-555.

Meyer L, Rabaud C, Ziegler O, May T \& Drouin P (1998) Protease inhibitors, diabetes mellitus and blood lipids. Diabetes Metabolism 24, 547-549.

Mulligan K, Grunfeld C, Hellerstein M, Neese R \& Schambelan M (1993) Anabolic effects of recombinant human growth hormone in patients with wasting associated with HIV infection. Journal of Clinical Endocrinology and Metabolism 77, 956-962.

Noor MA, Lo JC, Mulligan K, Shwarz JM, Halvorsen RA, Schambelan M \& Grunfeld C (2001) Absent HIV infection, Indinavir causes insulin resistance. AIDS 15, 11-18.

Nunez E \& Christeff N (1994) Steroid hormone, cytokine, lipid and metabolic disturbances in HIV infection. Baillieres Clinical Endocrinology and Metabolism 8, 803-824.

Pacht ER, Diaz P, Clanton T, Hart J \& Gaadek JE (1997) Serum vitamin $\mathrm{E}$ decreases in HIV-seropositive subjects over time. Journal of Laboratory and Clinical Medicine 130, 293-296.
Purnell J, Zambon A, Knopp R etal. (1999) Effect of Ritonavir on lipids and post-heparin lipase activities in normal subjects. AIDS 14, 51-57.

Roubenouff R, Weiss L, McDermott A, Heflin T, Cloutier G, Wood M \& Gorbach S (1999) A pilot study of exercise training to reduce trunk fat in adults with HIV-associated fat redistribution. AIDS 13, 1373-1375.

Sappey C, Leclercq P, Coudray C, Faure P, Micoud M \& Favier A (1994) Vitamin, trace element and peroxide status in HIV seropositive patients: asymptomatic patients present a severe betacarotene deficiency. Clinica Chimica Acta 230, 35-42.

Silva M, Skolnik PR, Gorbach SL, Spiegelman D, Wilson IB, Fernandez-Di Franco MG \& Knox TA (1998) The effect of protease inhibitors on weight and body composition in HIVinfected patients. AIDS 12, 1645-1651.

Sullivan AK, Feher MD, Nelson MR \& Gazzard BG (1998) Marked hypertriglyceridaemia associated with ritonavir therapy. AIDS 12, 1393-1395.

Taskinen MR (1995) Insulin resistance and lipoprotein metabolism. Current Opinion in Lipidology 6, 153-160.

Terrence Higgins Trust (2001) Terrence Higgins Trust factsheet. http://www.tht.org.uk/facts.htm.

Tikkanen MJ, Ojala J \& Helve E (1992) Long term use of Lovastatin in different types of hyperlipidaemia. Atherosclerosis 97, S27-S32.

Turcotte L, Srivastava A \& Chiasson J (1997) Fasting increases plasma membrane fatty acid-binding protein in red skeletal muscle. Molecular and Cellular Biochemistry 166, 153-158.

Ware LJ, Morlese J, Burdge G, Jackson AA, Gazzard B \& Wooton SA (2000) Differences in postprandial lipid metabolism in patients with PI-associated and NRTI-associated lipodystrophy. Antiviral Therapy 5, Suppl. 5, 13.

Ware LJ, Morlese J, Gazzard B, Burdge GC, Jackson AA \& Wooton SA (2001) Highly active anti-retroviral therapy (HAART) in HIV-1 positive men increases the retention time of dietary lipid within the circulation. Proceedings of the Nutrition Society 60, 31A.

Xiang S, Cianflone K, Kalant D \& Sniderman A (1999) Differential binding of triglyceride-rich lipoproteins to lipoprotein lipase. Journal of Lipid Research 40, 1655-1662.

Yarasheski K, Tebas P \& Stanerson B (2001) Resistance exercise training reduces hypertriglyceridaemia in HIV-infected men treated with antiviral therapy. Journal of Applied Physiology 90, 133-138. 